\title{
The Conversion of Excitation Energy into Generated Energy at the Resonant Frequencies of a Transparent Nonlinear Layer
}

\author{
Lutz Angermann",*, Vasyl Yatsyk², Mykola Yatsyk ${ }^{3}$ \\ ${ }^{1}$ Department of Mathematics, Clausthal University of Technology, Clausthal-Zellerfeld, Federal Republic of Germany \\ ${ }^{2}$ Department of Diffraction Theory and Diffraction Electronics, O. Ya. Usikov Institute for Radiophysics and Electronics of the \\ National Academy of Sciences of Ukraine, Kharkiv, Ukraine \\ ${ }^{3}$ Department of Electronic Computers, Kharkiv National University of Radio Electronics, Kharkiv, Ukraine \\ *corresponding author, E-mail: lutz . angermann@tu-claus thal. de
}

\begin{abstract}
An iterative algorithm is presented for analyzing the optimal resonant radiation properties of electromagnetic waves by cubically polarized nonlinear layers. The analysis is based on mathematical models for the rigorous treatment of the following problems: Self-consistent solution of both the system of boundary value problems of electrodynamics at resonant frequencies of excitation and generation, as well as the corresponding linearized eigenvalue problems with induced dielectric coefficients. The choice of the resonant excitation frequency of a nonlinear object in dependence on the real parts of the eigen frequencies of the spectral problems is discussed.

For the example of an absolutely transparent nonlinear layer in a linear approximation (with a linear component of the permittivity equal to one), the effect of converting the excitation field energy into the field energy of the generated third harmonic is demonstrated. The excitation frequency (basic frequency) is chosen such that the resonant generation frequency (the tripled frequency) coincides with the real part of the corresponding eigenvalue of the induced spectral problem. Here, with an increase of the amplitude of the incident field, the values of the scattered and generated energies in the reflection zone are insignificant. In this case, an effect of energy conversion can be observed in the transition zone: a decrease in the scattered energy and an increase in the generated one.
\end{abstract}

\section{Introduction}

The interest in the study of the properties of nonlinear objects has not diminished over the decades. This is evidenced by the results of many theoretical and experimental studies, among which we mention the works [1-7]. In this paper, we concentrate on the analysis of resonant properties inherent in layered, cubically polarizable media. It should be pointed out that, for a small amplitude of the irradiating field (that is if the generation of higher harmonics can be neglected) it is possible to describe a nonlinear medium by means of the simplest Kerr approximation. In such a situation, it is possible to analyze nonlinear layered structures as objects of resonant wave scattering only. In the general case of excitation by a packet of harmonic oscillations at multiple frequencies, the problem of numerical computation is reduced to a system of coupled boundary value problems with nonlinear Kerr-type permittivities induced at the multiple frequencies. In most cases, the excitation by a single harmonic field is considered, and the investigation is reduced to the rigorous solution of a nonlinear boundary value problem. An increase in the amplitude of the incident field leads to both scattering and generation of waves at multiple harmonics. Even in the case of excitation by a single plane wave, a multi-frequency system of coupled nonlinear boundary value problems with induced permittivities and source functions is generated. The analysis of the resonance properties of wave radiation by nonlinear layered structures is reduced to solving self-consistently a system of nonlinear Hammerstein integral equations or a system of boundary value problems of Sturm-Liouville type, both of which are equivalent to the original system of nonlinear boundary value problems [8-13].

The presented work continues our studies of the physical features of resonant radiation by an absolutely transparent nonlinear layer in a linear approximation with a linear component of the permittivity equal to one $[10,13]$. In the previous works, the effect of heavy conversion of excitation energy into generated energy of a transparent nonlinear layer was discovered. In this paper, we explain the physical mechanism of the mentioned effect. We show that it is due to the resonant properties of the generated oscillations, that is, the coincidence of the real part of the eigenvalue of the spectral problem at the frequency of the generated oscillations with the generation frequency.

The paper presents the numerical algorithm and the results of computations demonstrating the effect of optimal resonant conversion of the excitation energy into the generated energy. The dynamics of energetic characteristics and permittivities of the investigated processes of nonlinear resonant radiation is demonstrated.

Certain properties of the numerical method were checked computationally to confirm the validity of the proposed mathematical model and the results of the calculations obtained. 


\section{The dynamics of nonlinear resonance and the method of its analysis}

\subsection{The integral equation appraoch}

The problem of resonant radiation of waves by a nonlinear, nonmagnetic, isotropic, E-polarized

$$
\mathbf{E}=\left(E_{1}, 0,0\right)^{\top}, \mathbf{H}=\left(0, H_{2}, H_{3}\right)^{\top},
$$

cubically polarizable

$$
\mathbf{P}^{(\mathrm{NL})}=\left(P_{1}^{(\mathrm{NL})}, 0,0\right)^{\top},
$$

layered dielectric object (see Fig. 1) is investigated in a selfconsistent way, see $[8,13]$. The time dependency is of the form $\exp (-i n \omega t), \quad n \in \mathrm{N}=\{1,2,3, \ldots\}$. Here the dimensionless variables $x, y, z, t$ denote spatial-temporal coordinates such that the layer has the thickness $4 \pi \delta$, where $\delta>0 ; n \omega=n \kappa c$ are the circular frequencies, $n \kappa=2 \pi / \lambda_{n \kappa}$ are the frequency parameters; $\lambda_{n \kappa}$ are the incoming wave lengths; $c=\left(\varepsilon_{0} \mu_{0}\right)^{-1 / 2}, \operatorname{Im} c=0, \varepsilon_{0}$ and $\mu_{0}$ are the freespace permittivity and permeability, respectively.

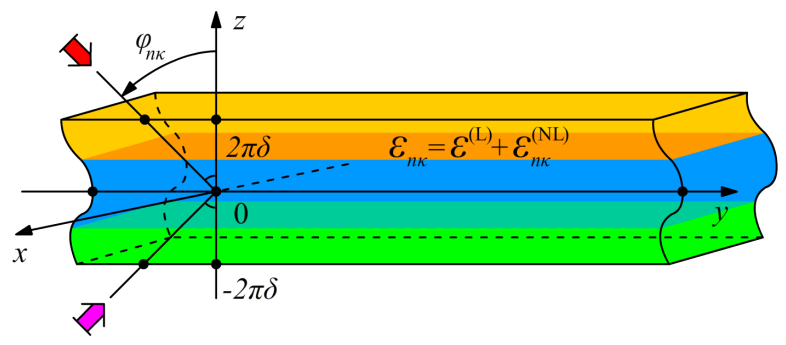

Figure 1: The plate-like structure with nonlinear layers.

It is presumed that the irradiating wave packet consists of an intense field at a frequency $\kappa$ and of weak fields at the multiple frequencies $2 \kappa$ and $3 \kappa$. Only the strong field is generating waves at the tripled frequency. Specifically, the irradiating wave packet has the form

$$
\left\{\bar{E}_{1}^{\text {inc }}(n \kappa ; y, z)\right\}_{n=1}^{3} \cup\left\{\underline{E}_{1}^{\text {inc }}(n \kappa ; y, z)\right\}_{n=1}^{3}
$$

With

$$
\begin{aligned}
& \left\{\begin{array}{l}
\bar{E}_{1}^{\text {inc }}(n \kappa ; y, z) \\
\underline{E}_{1}^{\text {inc }}(n \kappa ; y, z)
\end{array}\right\}= \\
& =\left\{\begin{array}{l}
a_{n \kappa}^{\text {inc }} \\
b_{n \kappa}^{\text {inc }}
\end{array}\right\} \exp \left[i\left(\Phi_{n \kappa} y \mp \Gamma_{n \kappa}(z \mp 2 \pi \delta)\right)\right], z^{>} \pm 2 \pi \delta .
\end{aligned}
$$

It acts onto the layered structure at angles of incidence

$$
\left\{\varphi_{n \kappa}, \pi-\varphi_{n \kappa}:\left|\varphi_{n \kappa}\right|<\pi / 2\right\}_{n=1}^{3}
$$

and with amplitudes $\left\{a_{n \kappa}^{\text {inc }}, b_{n \kappa}^{\text {inc }}\right\}_{n=1}^{3}$ at the frequencies $\{n \kappa\}_{n=1}^{3}$.

Here

$$
\Phi_{n \kappa}=n \kappa \sin \varphi_{n \kappa}, \Gamma_{n \kappa}=\left((n \kappa)^{2}-\Phi_{n \kappa}{ }^{2}\right)^{1 / 2}
$$

at $\operatorname{Re} \kappa \operatorname{Re} \Gamma_{n \kappa} \geq 0, \operatorname{Im} \Gamma_{n \kappa} \geq 0$

are the longitudinal and transverse propagation constants.

The structure is irradiated by a packet of planar stationary quasi-homogeneous (along the co-ordinate $y$ ) waves (1) satisfying a condition of phase synchronism (see $[10,13])$ :

$$
\begin{gathered}
\Phi_{n \kappa}=n \Phi_{\kappa}, \text { that is } \varphi_{n \kappa}=\varphi_{\kappa}, \\
n=1,2,3 .
\end{gathered}
$$

In such a situation the dielectric coefficients at the frequencies $n \kappa$ are homogeneous along the co-ordinate $y$ (see $[10,13])$. They have the form

$$
\begin{aligned}
& \varepsilon_{n \kappa}=\left\{1,|z|>2 \pi \delta ; \text { and } \varepsilon^{(\mathrm{L})}+\varepsilon_{n \kappa}^{(\mathrm{NL})},|z| \leq 2 \pi \delta\right\}, \\
& \varepsilon^{(\mathrm{L})}=1+4 \pi \chi_{11}^{(1)}(z), \\
& \varepsilon_{n \kappa}^{(\mathrm{NL})}=\alpha(z) \times \\
& \times\left[\sum_{m=1}^{3}\left|E_{1}(m \kappa ; y, z)\right|^{2}+\left\{\delta_{n}^{1} \frac{\left[E_{1}^{*}(\kappa ; y, z)\right]^{2}}{E_{1}(\kappa ; y, z)}+\right.\right. \\
& \left.\left.+\delta_{n}^{2} \frac{E_{1}^{*}(2 \kappa ; y, z)}{E_{1}(2 \kappa ; y, z)} E_{1}(\kappa ; y, z)\right\} E_{1}(3 \kappa ; y, z)\right], \\
& n=1,2,3,
\end{aligned}
$$

where

$$
\begin{aligned}
& E_{1}(n \kappa ; y, z)= \\
& =U(n \kappa ; z) \exp \left(i \Phi_{n \kappa} y\right)= \\
& =\chi(z-2 \pi \delta) \bar{E}_{1}^{\text {inc }}(n \kappa ; y, z)+ \\
& +\chi(-z-2 \pi \delta) \underline{E}_{1}^{\text {inc }}(n \kappa ; y, z)+ \\
& +E_{1}^{\text {scat/gen }}(n \kappa ; y, z)
\end{aligned}
$$

are the quasi-homogeneous (along the co-ordinate $y$ ) scattered/generated fields at the multiple frequencies $\{n \kappa\}_{n=1}^{3}$

$$
\alpha(z)=3 \pi \chi_{1111}^{(3)}(z)
$$

is the cubic susceptibility function of the nonlinear object; $\chi_{11}^{(1)}$ and $\chi_{1111}^{(3)}$ are the entries of the susceptibility tensors of the nonlinear medium;

$$
\chi(z)=\{0, z<0 ; \text { and } 1, z \geq 0\}
$$

is the Heaviside function; $\delta_{n}^{k}$ is Kronecker's symbol, and “*” is the symbol of complex conjugation.

The problem of interest can be described mathematically by a system of nonlinear boundary value problems or, equivalently, by the following system of onedimensional nonlinear Hammerstein integral equations for the unknown functions $U(n \kappa, \cdot) \in L_{2}(-2 \pi \delta, 2 \pi \delta)$, see [8$11,13]$, 


$$
\begin{aligned}
& U(n \kappa ; z)+\frac{i(n \kappa)^{2}}{2 \Gamma_{n \kappa}} \int_{-2 \pi \delta}^{2 \pi \delta} \exp \left(i \Gamma_{n \kappa}|z-\xi|\right) \times \\
& \times\left[1-\varepsilon_{n \kappa}\left(\xi, \alpha(\xi),\{U(m \kappa ; \xi)\}_{m=1}^{3}\right)\right] \times \\
& \times U(n \kappa ; \xi) d \xi= \\
& =\frac{i(n \kappa)^{2}}{2 \Gamma_{n \kappa}} \int_{-2 \pi \delta}^{2 \pi \delta} \exp \left(i \Gamma_{n \kappa}|z-\xi|\right) \alpha(\xi) \times \\
& \times\left[\delta_{n}^{1} U^{2}(2 \kappa ; \xi) U^{*}(3 \kappa ; \xi)+\delta_{n}^{3}\left\{\frac{1}{3} \times\right.\right. \\
& \left.\left.\times U^{3}(\kappa ; \xi)+U^{2}(2 \kappa ; \xi) U^{*}(\kappa ; \xi)\right\}\right] d \xi+ \\
& +\bar{U}^{\text {inc }}(n \kappa ; z)+\underline{U}^{\text {inc }}(n \kappa ; z), n=1,2,3,
\end{aligned}
$$

where

$$
\begin{aligned}
& \bar{U}^{\text {inc }}(n \kappa ; z)=a_{n \kappa}^{\text {inc }} \exp \left[-i \Gamma_{n \kappa}(z-2 \pi \delta)\right], \\
& \underline{U}^{\text {inc }}(n \kappa ; z)=b_{n \kappa}^{\text {inc }} \exp \left[i \Gamma_{n \kappa}(z+2 \pi \delta)\right]
\end{aligned}
$$

are functions defined by the excitation fields (1) with amplitudes $\left\{a_{n \kappa}^{\text {inc }}, b_{n \kappa}^{\text {inc }}\right\}_{n=1}^{3}$.

The fields (4) can be determined by solving the system (5) and can be represented in the form

$$
\begin{aligned}
& E_{1}(n \kappa ; y, z)=U(n \kappa ; z) \exp \left(i \Phi_{n \kappa} y\right)= \\
& =\left\{\begin{array}{r}
a_{n \kappa}^{\text {inc }} \exp \left\{i\left[\Phi_{n \kappa} y-\Gamma_{n \kappa}(z-2 \pi \delta)\right]\right\}+ \\
+a_{n \kappa}^{\text {scat/gen }} \exp \left\{i\left[\Phi_{n \kappa} y+\Gamma_{n \kappa}(z-2 \pi \delta)\right]\right\}, \\
\text { at } z>2 \pi \delta ; \\
U(n \kappa ; z) \exp \left(i \Phi_{n \kappa} y\right), \quad \text { at }|z| \leq 2 \pi \delta ; \\
b_{n \kappa}^{\text {inc }} \exp \left\{i\left[\Phi_{n \kappa} y+\Gamma_{n \kappa}(z+2 \pi \delta)\right]\right\}+ \\
+b_{n \kappa}^{\text {scat/gen }} \exp \left\{i\left[\Phi_{n \kappa} y-\Gamma_{n \kappa}(z+2 \pi \delta)\right]\right\}, \\
\text { at } z<-2 \pi \delta ;
\end{array}\right.
\end{aligned}
$$

using the formulas

$$
\begin{aligned}
& U(n \kappa ; 2 \pi \delta)=a_{n \kappa}^{\mathrm{inc}}+a_{n \kappa}^{\text {scat/gen }}, \\
& U(n \kappa ;-2 \pi \delta)=b_{n \kappa}^{\text {inc }}+b_{n \kappa}^{\text {scat/gen }}, \quad n=1,2,3 .
\end{aligned}
$$

\subsection{The coupled nonlinear algebraic equation system} and the induced eigenvalue problems

By using appropriate quadrature rules, the system of the nonlinear Hammerstein integral equations (5) can be transformed into a system of coupled nonlinear complex algebraic equations of the second kind $[12,13]$

$$
\begin{aligned}
& {\left[\mathbf{I}-\mathbf{B}_{n \kappa}\left(\mathbf{U}_{\kappa}, \mathbf{U}_{2 \kappa}, \mathbf{U}_{3 \kappa}\right)\right] \mathbf{U}_{n \kappa}=} \\
& =\delta_{n}^{1} \mathbf{C}_{\kappa}\left(\mathbf{U}_{2 \kappa}, \mathbf{U}_{3 \kappa}\right)+\delta_{n}^{3} \mathbf{C}_{3 \kappa}\left(\mathbf{U}_{\kappa}, \mathbf{U}_{2 \kappa}\right)+ \\
& +\overline{\mathbf{U}}_{n \kappa}^{\text {inc }}+\underline{\mathbf{U}}_{n \kappa}^{\text {inc }}, \quad n=1,2,3,
\end{aligned}
$$

and to the induced eigenvalue problems in the complex region of the frequency parameters, see Figs. 2, 3 and [13, 14],

$$
\begin{gathered}
\left\{\begin{array}{l}
f_{n \kappa}\left(\kappa_{n}\right)=\operatorname{det}\left[\mathbf{I}-\mathbf{B}_{n \kappa}\left(\kappa_{n}\right)\right]=0, \\
{\left[\mathbf{I}-\mathbf{B}_{n \kappa}\left(\kappa_{n}\right)\right] \mathbf{U}_{\kappa_{n}}=\mathbf{0} ;}
\end{array}\right. \\
\quad \kappa \equiv \kappa^{\mathrm{inc}} ; \quad \kappa_{n} \in \Omega_{n \kappa} \subset \mathrm{H}_{n \kappa} .
\end{gathered}
$$
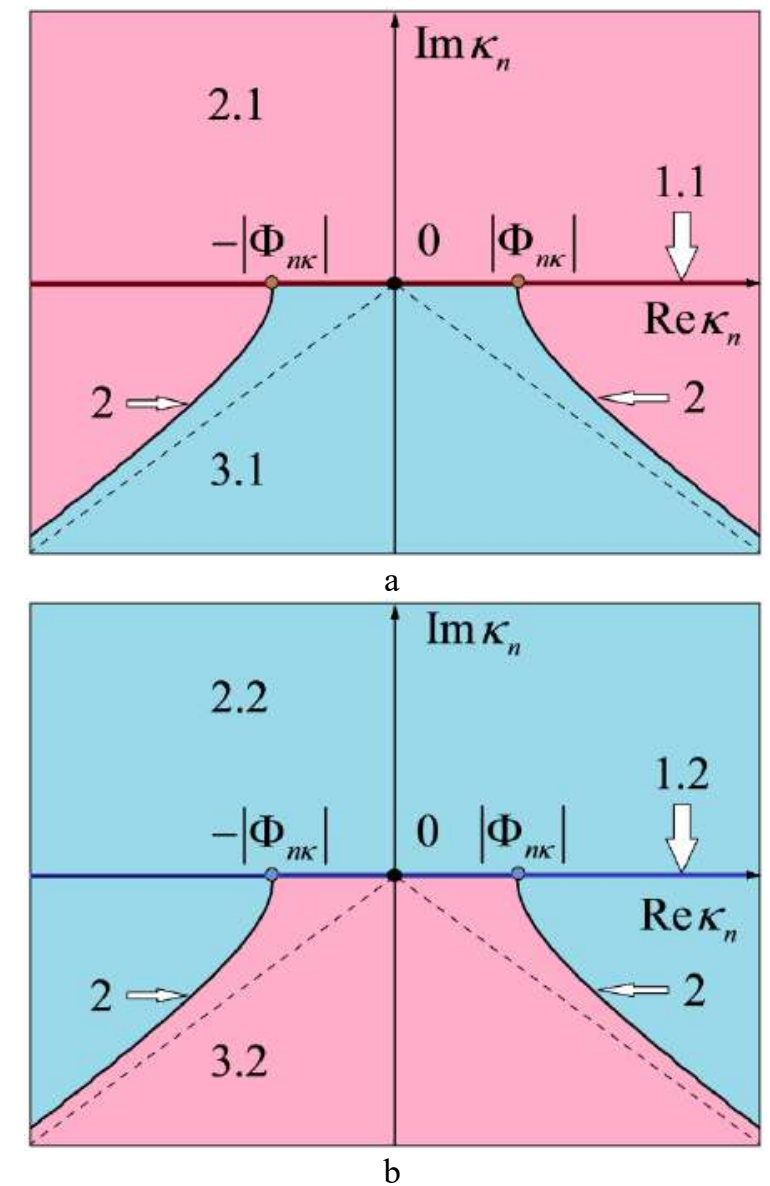

Figure 2: (a) Physical and (b) non-physical sheets $\mathrm{H}_{n \kappa}$, $n=1,2,3$. Branch points $\kappa_{n}^{ \pm}= \pm\left|\Phi_{n \kappa}\right|$; cuts

(a) and (b) $2 \ldots\left(\operatorname{Re} \kappa_{n}\right)^{2}-\left(\operatorname{Im} \kappa_{n}\right)^{2}-\Phi_{n \kappa}{ }^{2}=0, \operatorname{Im} \kappa_{n} \leq 0$;

(a) $1.1 \ldots \operatorname{Re} \kappa_{n} \operatorname{Re} \Gamma_{\kappa_{n}} \leq 0, \operatorname{Im} \Gamma_{\kappa_{n}} \geq 0\left(\operatorname{Im} \kappa_{n}=0\right)$;

(a) $2.1 \ldots \operatorname{Re} \kappa_{n} \operatorname{Re} \Gamma_{\kappa_{n}} \leq 0, \operatorname{Im} \kappa_{n} \operatorname{Im} \Gamma_{\kappa_{n}} \leq 0\left(\operatorname{Im} \kappa_{n} \neq 0\right)$;

(a) $3.1 \ldots \operatorname{Re} \kappa_{n} \operatorname{Re} \Gamma_{\kappa_{n}} \leq 0, \operatorname{Im} \kappa_{n} \operatorname{Im} \Gamma_{\kappa_{n} \geq} \leq 0$.

Here

$$
\left\{\left\{z_{l}\right\}_{l=1}^{N}: z_{1}=-2 \pi \delta<\ldots<z_{l}<\ldots<z_{N}=2 \pi \delta\right\}
$$

are the interpolation nodes. In problem (7),

$$
\mathbf{U}_{n \kappa}=\left(U\left(n \kappa ; z_{1}\right), \ldots, U\left(n \kappa ; z_{N}\right)\right)^{\top}
$$


denote the vectors of the solution to be determined; $\mathbf{I}$ is the identity matrix; $\overline{\mathbf{U}}_{n \kappa}^{\text {inc }}$ and $\underline{\mathbf{U}}_{n \kappa}^{\text {inc }}$ are the amplitude vectors defined by the incident waves; $\mathbf{B}_{n \kappa}\left(\mathbf{U}_{\kappa}, \mathbf{U}_{2 \kappa}, \mathbf{U}_{3 \kappa}\right) \equiv \mathbf{B}_{n \kappa}\left(\left\{\mathbf{U}_{p \kappa}\right\}_{p=1}^{3}\right) \quad$ are matrix-valued nonlinear functions; $\mathbf{C}_{\kappa}\left(\mathbf{U}_{2 \kappa}, \mathbf{U}_{3 \kappa}\right), \mathbf{C}_{3 \kappa}\left(\mathbf{U}_{\kappa}, \mathbf{U}_{2 \kappa}\right)$ are the right-hand side vectors determined by the particular choice of the quadrature formula. In problem (8), $\Omega_{n \kappa}$ is the discrete set of the eigenvalues $\kappa_{n}$ to be determined; $\mathrm{H}_{n \kappa}$ are two-sheeted Riemann manifolds the sheets of which uniquely specify the functions of the transverse propagation

$$
\Gamma_{\kappa_{n}}\left(\kappa_{n}, \Phi_{n \kappa}\right)=\left(\kappa_{n}^{2}-\Phi_{n \kappa}{ }^{2}\right)^{1 / 2}
$$

(depending on the complex eigenvalues $\kappa_{n}$ at the known real values of the longitudinal propagation constants $\left.\Phi_{n \kappa}=n \kappa \sin \varphi_{n \kappa}\right)$, cf. Fig. 2;

$$
\mathbf{U}_{\kappa_{n}}=\left(U\left(\kappa_{n} ; z_{1}\right), \ldots, U\left(\kappa_{n} ; z_{N}\right)\right)^{\top}
$$

are the vectors of the values of the unknown nontrivial solution at the nodes in the layer corresponding to the eigenfrequencies $\kappa_{n} ; \mathbf{B}_{n \kappa}\left(\kappa_{n}\right)=\mathbf{B}_{n \kappa}\left(\kappa_{n} ;\left\{\mathbf{U}_{p \kappa}\right\}_{p=1}^{3}\right)$ are matrices depending on the given vectors $\left\{\mathbf{U}_{p \kappa}\right\}_{p=1}^{3}$, see (7) and (8).

In the computational experiments, we use the structure of the Riemann manifolds $\mathrm{H}_{n \kappa}$ depicted in Fig. 2. These surfaces $\mathrm{H}_{n \kappa}$ (see Fig. 2) are two-sheeted with real algebraic branch points of second order $\kappa_{n}^{ \pm}: \Gamma_{\kappa_{n}}\left(\kappa_{n}^{ \pm}, \Phi_{n \kappa}\right)=0$ (i.e. $\kappa_{n}^{ \pm}= \pm\left|\Phi_{n \kappa}\right|, n=1,2,3$ ) and cuts along the lines no. 2. The physical and non-physical sheets $\mathrm{H}_{n \kappa}$ defined by one and the same configuration of the cuts no. 2 will be called dual. Those submanifolds of the dual sheets $\mathrm{H}_{n \kappa}$ where the values $\left\{\operatorname{Re} \kappa_{n} \operatorname{Re} \Gamma_{\kappa_{n}}, \operatorname{Im} \kappa_{n} \operatorname{Im} \Gamma_{\kappa_{n}}\right\}$ have identical signs are marked on the physical and non-physical sheets of the surface $\mathrm{H}_{n \kappa}$ with the same color, see Fig. 2. On the real axis $\operatorname{Re} \kappa_{n}$ of the first physical sheet, see Fig. 2 (a), the values $\left\{\operatorname{Re} \Gamma_{\kappa_{n}}, \operatorname{Im} \Gamma_{\kappa_{n}}\right\}$ correspond to the prescription of the physically motivated radiation condition no. 1.1. The analytical continuation of $\Gamma_{\kappa_{n}}$ into the complex plane of eigenvalues $\kappa_{n}$, taking into account the requirement no. 1.1 and the cuts no.2, uniquely determines the values of $\left\{\operatorname{Re} \Gamma_{\kappa_{n}}, \operatorname{Im} \Gamma_{\kappa_{n}}\right\}$ on the first physical sheet $\mathrm{H}_{n \kappa}$ under the conditions no. 2.1 and 3.1, see. Fig. 2 (a). The second nonphysical sheet differs from the first physical sheet in the replacement of the signs of the pair $\left\{\operatorname{Re} \Gamma_{\kappa_{n}}, \operatorname{Im} \Gamma_{\kappa_{n}}\right\}$ by the opposite ones, see. nos. 1.2, 2.2 and 2.3 in Fig. 2 (b). In this case, the requirement no. 1.2 plays the role of an excitation condition.

We mention that the cuts no. 2 can be given on a sheet of the Riemann manifolds $\mathrm{H}_{n \kappa}, n=1,2,3$, by arbitrary continuous curves located in the third and fourth quadrants of the complex plane and connecting the branch points with the point at infinity, see $[13,14]$.

Examples of physical sheets of the Riemann manifolds $\mathrm{H}_{n \kappa}, n=1,2,3$ with different configurations of cuts along the coordinate axes are shown in Fig. 3.

The affiliation of the frequency spectral parameter $\kappa_{n} \in \Omega_{n \kappa} \subset \mathrm{H}_{n \kappa}$ (i.e., the pair $\left(\operatorname{Re} \kappa_{n}, \operatorname{Im} \kappa_{n}\right)$ ) to the physical or non-physical sheets of the surface $\mathrm{H}_{n \kappa}$ with different configurations of the cuts can be checked by the fulfillment of the conditions no. 2.1, 3.1 and 2.2, 3.2 defining the submanifolds, see Figs. 2 and 3.
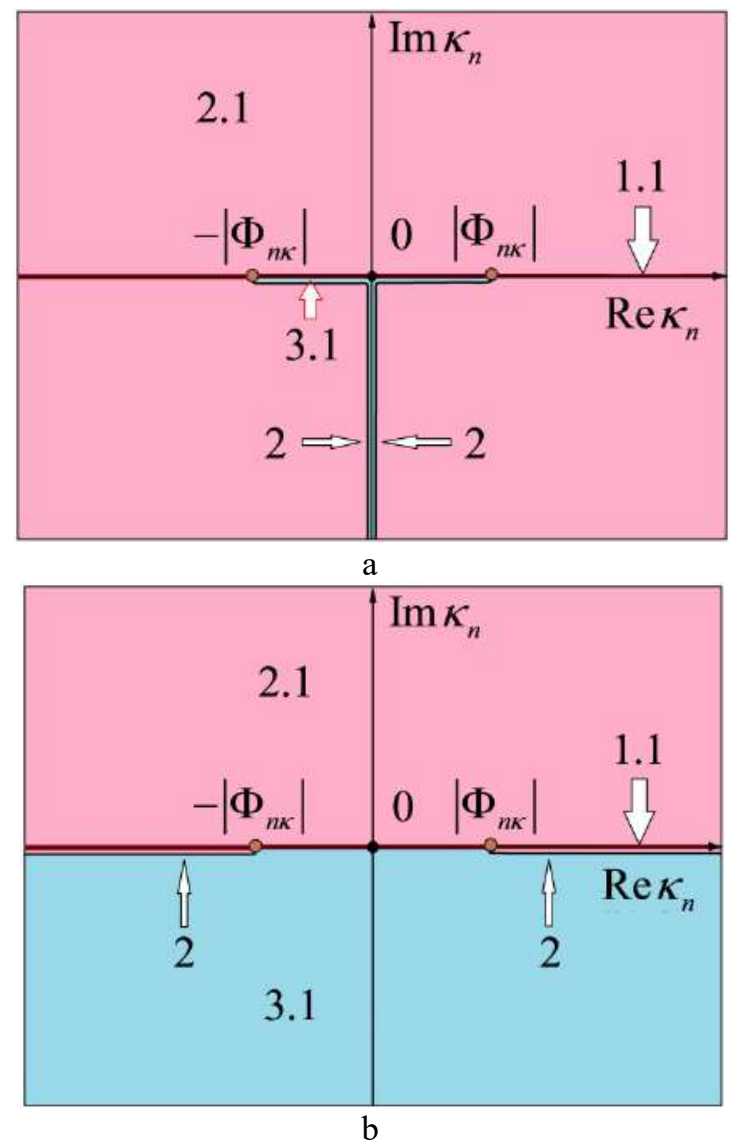

Figure 3: Variants of physical sheets of the Riemann manifolds $\mathrm{H}_{n \kappa}, n=1,2,3$, with the branch points $\kappa_{n}^{ \pm}= \pm\left|\Phi_{n \kappa}\right|$ and $2 \ldots$ cuts along (a) segments of the real and imaginary axes, (b) imaginary semi-axes; submanifolds: $\quad 1.1 \ldots \operatorname{Re} \kappa_{n} \operatorname{Re} \Gamma_{\kappa_{n}} \geq 0, \quad \operatorname{Im} \Gamma_{\kappa_{n}} \geq 0$ $\left(\operatorname{Im} \kappa_{n}=0\right) \quad-\quad$ radiation condition; $2.1 \ldots \operatorname{Re} \kappa_{n} \operatorname{Re} \Gamma_{\kappa_{n}} \geq 0, \operatorname{Im} \kappa_{n} \operatorname{Im} \Gamma_{\kappa_{n}} \geq 0\left(\operatorname{Im} \kappa_{n} \neq 0\right)$;

$3.1 \ldots \operatorname{Re} \kappa_{n} \operatorname{Re} \Gamma_{\kappa_{n}} \leq 0, \operatorname{Im} \kappa_{n} \operatorname{Im} \Gamma_{\kappa_{n}} \leq 0$.

For example, consider the third or fourth quadrant of the surface $\mathrm{H}_{n \kappa}$ shown in Fig. 2. Let a branch of the dispersion curve $\kappa_{n}(\tau) \in \Omega_{n \kappa}(\tau) \subset \mathrm{H}_{n \kappa}$ (with some parameter $\tau$ ) go 
from a physical sheet (region no. 2.1) to a non-physical sheet (region no. 3.2). Such a behaviour of the curve $\kappa_{n}(\tau)$ (due to the coincidence of the conditions to the regions no. 2.1 and 3.2, i.e. $\operatorname{Re} \kappa_{n} \operatorname{Re} \Gamma_{\kappa_{n}} \geq 0, \operatorname{Im} \kappa_{n} \operatorname{Im} \Gamma_{\kappa_{n}} \geq 0$, $\left.\left(\operatorname{Im} \kappa_{n} \neq 0\right)\right)$ is accompanied by the conservation of the signs of $\left\{\operatorname{Re} \Gamma_{\kappa_{n}}, \operatorname{Im} \Gamma_{\kappa_{n}}\right\}$ when changing from one sheet to the other. The same dispersion curves $\kappa_{n}(\tau)$ completely belong to single sheets of the surfaces $\mathrm{H}_{n \kappa}$ defined by different variants of the cuts no. 2 in Fig. 3, namely to the physical sheet $\mathrm{H}_{n \kappa}$ shown in Fig. 3 (a), and to the nonphysical sheet $\mathrm{H}_{n \kappa}$, dual to the physical sheet $\mathrm{H}_{n \kappa}$, shown in Fig. 3(b). Here also the values of the quantities $\left\{\operatorname{Re} \Gamma_{\kappa_{n}}, \operatorname{Im} \Gamma_{\kappa_{n}}\right\}$ are determined by the identical conditions no. 2.1 and 3.2.

\subsection{The algorithm of the dynamics of resonant processes}

The study of the behaviour of resonant wave radiation for the variation of the excitation amplitudes at frequencies in the vicinity of the induced eigenvalues of the nonlinear layered object under consideration is carried out by the help of the algorithm [12]:

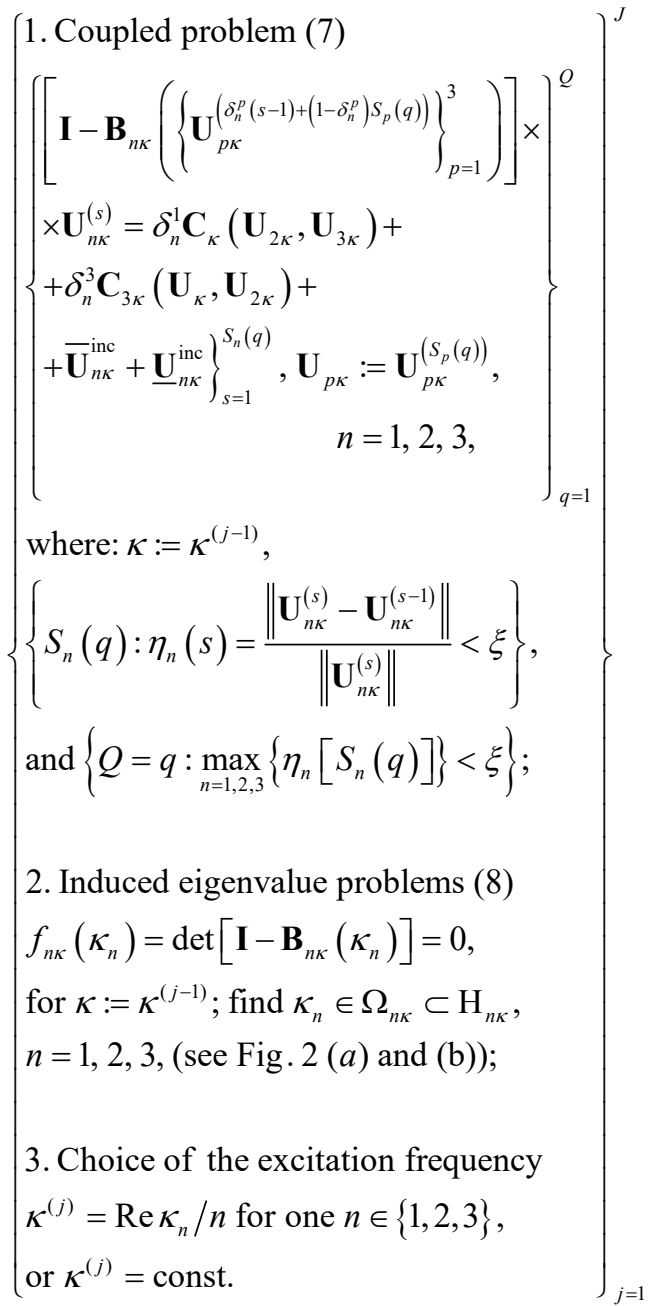

The stopping criterion for (9) is

$$
\left\{J=j \in \mathrm{N}: \eta(j)=\frac{\left\|\kappa^{(j)}-\kappa^{(j-1)}\right\|}{\left\|\kappa^{(j)}\right\|}<\xi\right\},
$$

where $\xi>0$ is a given value of the relative computing error.

In the scheme (9), the following steps are successively performed: the solution of the coupled problem (by means of the scheme (6)); the solution of the induced eigenvalue problems (7); the selection of the excitation frequency from the values given in the last block of the algorithm (9).

To describe the radiation properties of the nonlinear object, we use the following notation:

$$
\begin{aligned}
& R_{n \kappa}^{+}=\left|a_{n \kappa}^{\text {scatgen }}\right|^{2} / \sum_{s=1}^{3}\left(\left|a_{s \kappa}^{\text {inc }}\right|^{2}+\left|b_{s \kappa}^{\text {inc }}\right|^{2}\right), \\
& R_{n \kappa}^{-}=\left|b_{n \kappa}^{\text {scat } / \text { gen }}\right|^{2} / \sum_{s=1}^{3}\left(\left|a_{s \kappa}^{\text {inc }}\right|^{2}+\left|b_{s \kappa}^{\text {inc }}\right|^{2}\right) .
\end{aligned}
$$

The quantities $R_{n \kappa}^{ \pm}$characterize the proportions of the radiated energy to the total excitation energy.

The fractions of the radiated energy at the frequencies $n \kappa$ correspond to the quantities

$$
W_{n \kappa}=\left|a_{n \kappa}^{\text {scatgen }}\right|^{2}+\left|b_{n \kappa}^{\text {scatgen }}\right|^{2} .
$$

The proportions of the energy of the third harmonic to the energy radiated by the first harmonic and to the total energy are defined as

$$
\begin{aligned}
& W_{\{3: 1 ; \kappa\}}=W_{3 \kappa} / W_{\kappa}, \\
& W_{\{3: 123 ; \kappa\}}=W_{3 \kappa} / \sum_{n=1}^{3} W_{n \kappa} .
\end{aligned}
$$

The behaviour of the relative Q-factor

$$
Q_{\{1: 3 ; \kappa\}}=Q_{\kappa_{1} ; \kappa} / Q_{\kappa_{3} ; 3 \kappa}
$$

of eigen waves is of interest [13, 15-17].

Here

$$
Q_{\kappa_{n} ; n \kappa}=\left.\left\{-\operatorname{Re} \kappa_{n} /\left(2 \operatorname{Im} \kappa_{n}\right)\right\}\right|_{n \kappa}
$$

are the Q-factors of the waves with the eigenvalues $\kappa_{n} \in \Omega_{n \kappa} \subset \mathrm{H}_{n \kappa}$ of the corresponding eigenvalue problems induced at the frequencies $n \kappa, n=1,2,3$, see [13, 14].

\section{Results and discussion}

\subsection{Energetic properties of resonant scattering and generation}

Based on the algorithm (9) numerical investigations of the resonant properties of a single, absolutely transparent (in the linear approximation) nonlinear layer with the parameters:

$$
\left\{\left\{\varepsilon^{(\mathrm{L})}(z), \alpha(z)\right\}:\left\{\varepsilon^{(\mathrm{L})}=1, \alpha=+0.01\right\}, \delta=0.5\right\}
$$

were carried out (see (3)). The incident field acts in normal direction $\varphi_{n \kappa}=0^{0}, \quad n=1,2,3$, with amplitudes $a_{\kappa}^{\text {inc }} \neq 0$, $a_{2 \kappa}^{\text {inc }}=a_{3 \kappa}^{\text {inc }}=0, b_{\kappa}^{\text {inc }}=b_{2 \kappa}^{\text {inc }}=b_{3 \kappa}^{\text {inc }}=0$, and with an excitation frequency being equal to the corresponding resonant 
generation frequency of the third harmonic, i.e. $3 \kappa=\operatorname{Re} \kappa_{3}$ $(\operatorname{see}(9))$.

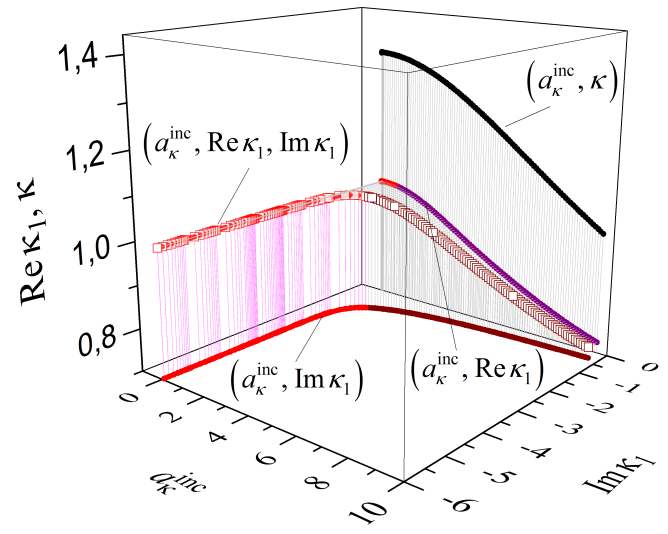

a

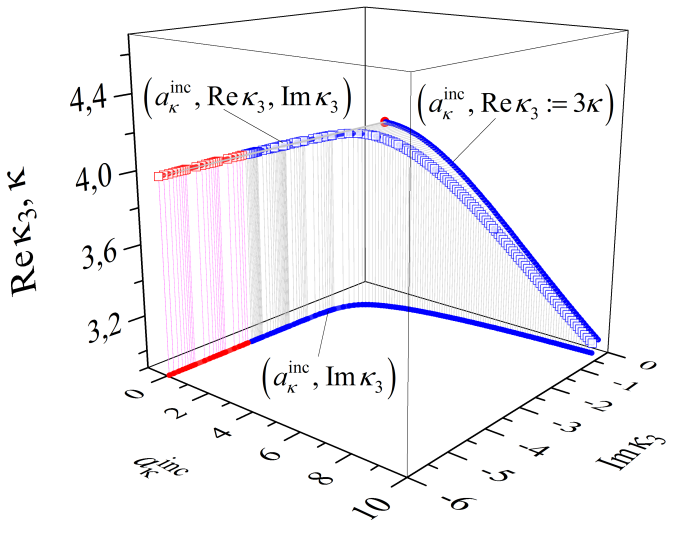

$\mathrm{b}$

Figure 4: The eigen frequency branches $\left(a_{\kappa}^{\text {inc }}, \operatorname{Re} \kappa_{n}, \operatorname{Im} \kappa_{n}\right)$ at $\kappa=\operatorname{Re} \kappa_{3} / 3$ and their projections onto the co-ordinate planes (a) for $n=1$ and (b) for $n=3$. The curves (a) of the excitation frequencies $\left(a_{\kappa}^{\text {inc }}, \kappa\right)$ and $(b)$ of the generation frequencies $\left(a_{\kappa}^{\mathrm{inc}}, \operatorname{Re} \kappa_{3}=: 3 \kappa\right)$.

The computational results obtained using the iterative algorithm (9) in the amplitude range $a_{\kappa}^{\text {inc }} \in\left[1.1 \cdot 10^{-7}, 9.6\right]$ are presented in Figs. 4-7. The calculation of the frequency characteristics $\quad \kappa_{n}\left(a_{\kappa}^{\text {inc }}\right)=\operatorname{Re} \kappa_{n}\left(a_{\kappa}^{\text {inc }}\right)+i \operatorname{Im} \kappa_{n}\left(a_{\kappa}^{\text {inc }}\right) \in$ $\Omega_{n \kappa}\left(a_{\kappa}^{\text {inc }}\right) \subset \mathrm{H}_{n \kappa}, n=1,3$, of the induced spectral problems (in the second block (9)) was carried out using the Riemann manifolds presented in Fig. 2. The parts of the eigen frequency branches $\left(a_{\kappa}^{\text {inc }}, \operatorname{Re} \kappa_{n}, \operatorname{Im} \kappa_{n}\right), n=1,3$, and their projections onto the coordinate planes belonging to different sheets of the Riemann manifolds $\mathrm{H}_{n \kappa}, n=1,3$, are marked in Fig. 4 by different colors. This also applies to the curves of the spectral characteristics $\operatorname{Re} \kappa_{n}\left(a_{\kappa}^{\text {inc }}\right), \operatorname{Im} \kappa_{n}\left(a_{\kappa}^{\text {inc }}\right)$, $n=1,3$, in Fig. 5 . Thus, the curve of the eigen frequency branch $\quad \kappa_{1}\left(a_{\kappa}^{\text {inc }}\right) \quad$ at $\quad a_{\kappa}^{\text {inc }} \in\left[1.1 \cdot 10^{-7}, 0.86\right) \quad$ and $\left[1.1 \cdot 10^{-7}, 0.86\right]$ lies on the non-physical and physical sheet, respectively, of the surface $\mathrm{H}_{\kappa}$, see Figs. 4 (a), 5 . Similarly, the branch $\kappa_{3}\left(a_{\kappa}^{\text {inc }}\right)$ at $a_{\kappa}^{\text {inc }} \in\left[1.1 \cdot 10^{-7}, 7 \cdot 10^{-5}\right)$ and $\left[7 \cdot 10^{-5}, 0.86\right]$ is located on the non-physical and physical sheet, respectively, of the surface $\mathrm{H}_{3 \kappa}$, see Figs. 4 (b), 5. In Figs. 4, 5 the graphs of the excitation frequencies $\left(a_{\kappa}^{\text {inc }}, \kappa\right), \kappa:=\operatorname{Re} \kappa_{3}\left(a_{\kappa}^{\text {inc }}\right) / 3$ defined in the third block of the algorithm (9) (see Figs. 4 (a), 5) and the graphs of the generation frequencies $\left(a_{\kappa}^{\text {inc }}, \operatorname{Re} \kappa_{3}=: 3 \kappa\right)$, $\operatorname{Re} \kappa_{3}\left(a_{\kappa}^{\text {inc }}\right)$ (see Figs. 4 (b), 5). are depicted.

Fig. 6, along with the energetic characteristics obtained by solving the self-consistent problem in the first block of the algorithm (9), also shows branches of the Q-factors $Q_{\kappa_{n} ; n \kappa}\left(a_{\kappa}^{\text {inc }}\right)$ for $n=1,3$ (see Fig. $6(\mathrm{a})$ ) and of the relative Q-factors $Q_{\{1: 3 ; \kappa\}}\left(a_{\kappa}^{\text {inc }}\right)$ (see Fig. $6(\mathrm{~b})$ ) corresponding to the eigen frequency branches depicted in the Figs. 4, 5 above. For very small values of the amplitudes $a_{\kappa}^{\text {inc }}$ of the incident field, the eigen frequencies with large negative values $\operatorname{Im} \kappa_{n}, n=1,3$, correspond to small Q-factors $Q_{\kappa_{n} ; n \kappa}\left(a_{\kappa}^{\text {inc }}\right)$, see Figs. 4, 5 .

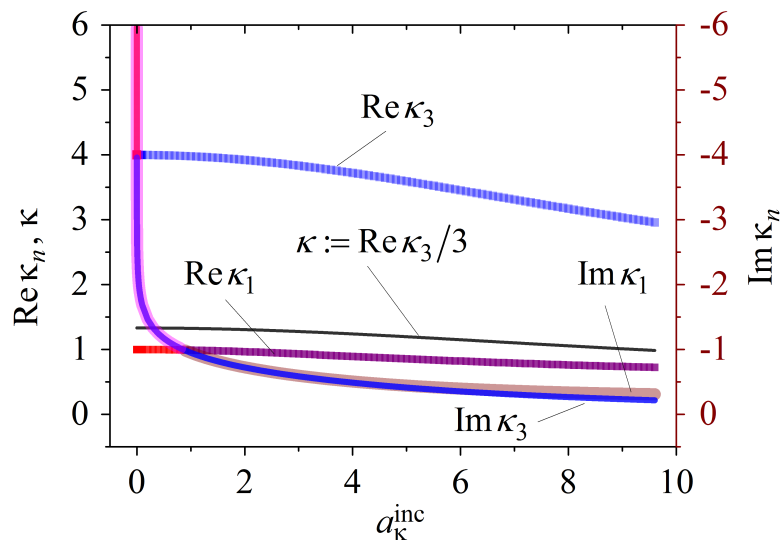

Figure 5: The curve of the excitation frequencies $\kappa:=\operatorname{Re} \kappa_{3}\left(a_{\kappa}^{\text {inc }}\right) / 3$ and the curves of the spectral characteristics $\operatorname{Re} \kappa_{n}\left(a_{\kappa}^{\mathrm{inc}}\right), \operatorname{Im} \kappa_{n}\left(a_{\kappa}^{\mathrm{inc}}\right)$ for $n=1,3$.

The dynamics of the relative quality factor $Q_{\{1: 3 ; \kappa\}}\left(a_{\kappa}^{\text {inc }}\right)$ is interesting, too. The monotone decrease of this quantity with growing amplitude of the irradiating field at the resonant excitation frequencies, determined by the last block in algorithm (9), is accompanied by an increase in the generating ability of the nonlinear object, see Fig. 6 (b).

In the case under consideration, an increase in the 


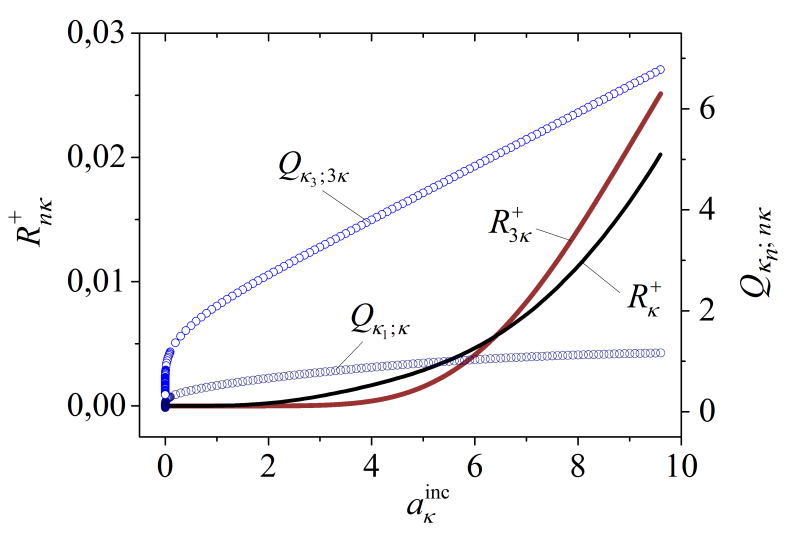

a

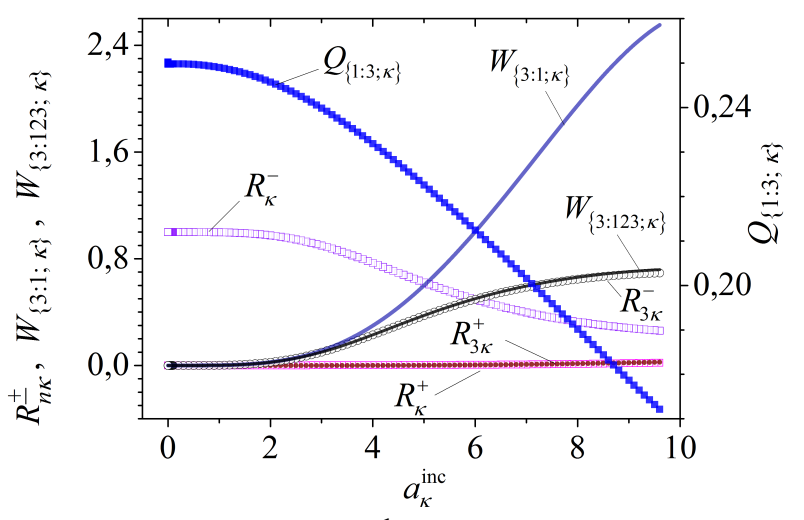

$\mathrm{b}$

Figure 6: Curves at the excitation frequencies $\kappa=\operatorname{Re} \kappa_{3}\left(a_{\kappa}^{\text {inc }}\right) / 3$ of the induced nonlinear layer: (a) the energetic characteristics $R_{n \kappa}^{+}\left(a_{\kappa}^{\text {inc }}\right)$ and the Q-factors $Q_{\kappa_{n} ; n \kappa}\left(a_{\kappa}^{\text {inc }}\right)$ for $n=1,3 ;$ (b) the energetic characteristics $R_{n \kappa}^{ \pm}\left(a_{\kappa}^{\text {inc }}\right)$ for $n=1,3, W_{\{3: 1 ; \kappa\}}\left(a_{\kappa}^{\text {inc }}\right), W_{\{3: 123 ; \kappa\}}\left(a_{\kappa}^{\text {inc }}\right)$ and the relative Q-factors $Q_{\{1: 3 ; \kappa\}}\left(a_{\kappa}^{\text {inc }}\right)$.

incident amplitude $a_{\kappa}^{\text {inc }}$ is accompanied by a slight increase in the values of the radiated energies $R_{\kappa}^{+}\left(a_{\kappa}^{\text {inc }}\right)$ and $R_{3 \kappa}^{+}\left(a_{\kappa}^{\text {inc }}\right)$, resp., in the reflection zone. The maximum values of these quantities for $a_{\kappa}^{\text {inc }}=9.6$ do not exceed $2.1 \%$ and $2.52 \%$, resp. (see Fig. 6). In the transition zone, an effect of energy conversion can be observed: a decrease in the scattered energy $R_{\kappa}^{-}\left(a_{\kappa}^{\text {inc }}\right)$ to $26.125 \%$ and an increase in the generated energy $R_{3 \kappa}^{-}\left(a_{\kappa}^{\text {inc }}\right)$ up to $69.338 \%$ for $a_{\kappa}^{\text {inc }}=9.6$, see Fig. $\left.6(\mathrm{~b})\right)$. At the same time, for the amplitude $a_{\kappa}^{\text {inc }}=9.6$, the fraction $W_{\{3: 1 ; \kappa\}}\left(a_{\kappa}^{\text {inc }}\right)$ of the total energy dissipated to the third harmonic exceeds by 2.55247 times the energy dissipated to the first harmonic, and the total generated energy $W_{\{3: 123 ; \kappa\}}\left(a_{\kappa}^{\text {inc }}\right)$ amounts to $71.851 \%$ of the total radiated energy.

In Fig. 7 the surfaces of the absolute values $\left|U_{\kappa}\right|$ and $\left|U_{3 \kappa}\right|$ of the amplitudes of the scattered and generated fields, resp., in the nonlinear layer at the scattering and generation frequencies $\kappa=\operatorname{Re} \kappa_{3}\left(a_{\kappa}^{\text {inc }}\right) / 3$ and $3 \kappa$ are shown, cf. (6). The surfaces of the real $\operatorname{Re} \varepsilon_{n \kappa}$ and imaginary $\operatorname{Im} \varepsilon_{n \kappa}$ parts of the nonlinear permittivities (3) induced by the fields (6) are also depicted. The results were obtained using the self-consistent analysis of the systems of nonlinear problems, see the first block in algorithm (9).

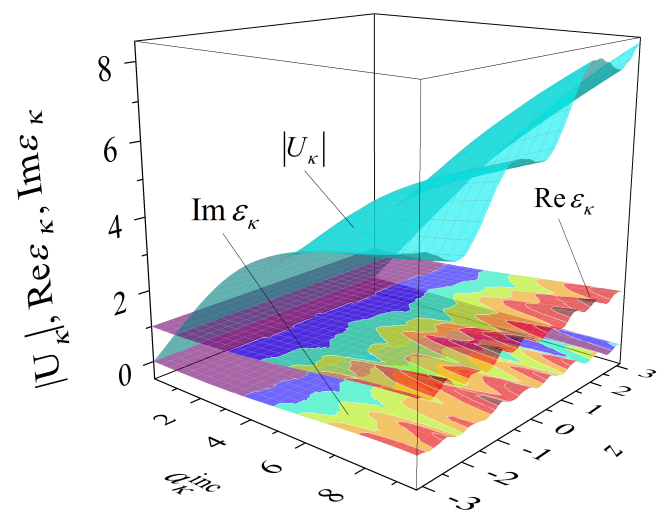

a

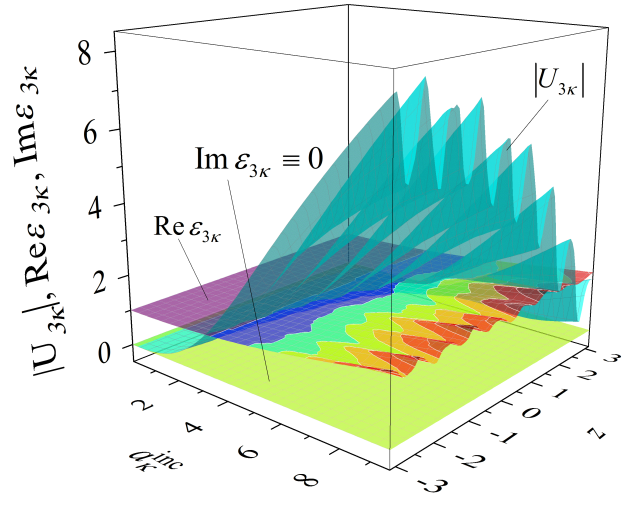

b

Figure 7: The amplitude characteristics $\left|U_{n \kappa}\right|$ and induced permittivities $\operatorname{Re} \varepsilon_{n \kappa}, \quad \operatorname{Im} \varepsilon_{n \kappa}$ for $n=1,3$ at (a) the scattering frequency $\kappa=\operatorname{Re} \kappa_{3}\left(a_{\kappa}^{\text {inc }}\right) / 3$, (b) the generation frequency $3 \kappa=\operatorname{Re} \kappa_{3}\left(a_{\kappa}^{\text {inc }}\right)$.

In the parameter range

$$
\left\{\left\{a_{\kappa}^{\text {inc }}, z\right\}: a_{\kappa}^{\text {inc }} \in\left[1.1 \cdot 10^{-7}, 9.6\right], z \in[-2 \pi \delta, 2 \pi \delta]\right\}
$$

under study, with an increase in the amplitude $a_{\kappa}^{\text {inc }}$ of the 
excitation field, a more pronounced resonance behaviour of the amplitude characteristics $\left|U_{\kappa}\right|$ and $\left|U_{3 \kappa}\right|$ of the radiated fields, resp., is observed in the $z$-direction (i.e. along the height of the nonlinear layer). In this case the layer is converted into a resonant layered structure, see Fig. 7.

Variations of the real parts of the permittivities are observed in the range $1 \leq \operatorname{Re} \varepsilon_{n \kappa} \leq 1.87979, n=1,3$, see Fig. 7. The imaginary part of the permittivity at the scattering frequency $\kappa$ is non-negative, it takes values in the range $0 \leq \operatorname{Im} \varepsilon_{\kappa} \leq 0,44146$, see Fig. 7 (a). This behaviour of $\operatorname{Im} \varepsilon_{\kappa}$ is due to the manifestation of the effect of energy conversion of the incident field into the generated one, that is, the energy expended by the third harmonic field. At the generation frequency $3 \kappa$ we have that $\operatorname{Im} \varepsilon_{3 \kappa} \equiv 0$, see Fig. 7 (b). This is due to our assumption about the weakness of the generated third-harmonic field. Such a field is involved in the generation process, but does not lead to the generation of new higher harmonics.

\subsection{Specifics in the computations of nonlinear resonant structures}

This section discusses criteria for evaluating the computational features of resonant radiation processes by nonlinear layered objects.

The calculations were performed with double precision in the standard arithmetic of complex numbers, the corresponding machine epsilon is equal to $\varepsilon_{\text {mash }}=2^{-52} \approx 2.22045 \cdot 10^{-16}$. This means that the number of significant decimal digits in the mantissa is not larger than 16.

The analysis of resonant processes is carried out on the basis of the iterative algorithm (9). The systems of nonlinear algebraic equations in the self-consistent problem (block 1 in algorithm (9)) and the systems of linear algebraic equations for the linearized spectral problems (block 2 in algorithm (9)) are derived from the systems of Hammerstein and Fredholm integral equations of the second kind by use of a composite closed Newton-Cotes' rule with 7 nodes per subinterval (Weddle's rule).

In the range of parameters under investigation, equation systems of dimension 43 were considered. The relative error of the algorithm (9) did not exceed the value $\xi<10^{-7}$ (see (10)).

In the considered case of a non-absorbing medium (where $\operatorname{Im} \varepsilon^{(\mathrm{L})} \equiv 0$ ), the energy balance law [13]

$$
\sum_{n=1}^{3}\left(R_{n \kappa}^{+}+R_{n \kappa}^{-}\right)=1 \text { for } \operatorname{Im} \varepsilon^{(\mathrm{L})} \equiv 0
$$

was verified numerically. In the numerical experiments, the magnitude of the energy balance error

$$
W^{(\text {Error })}=1-\sum_{n=1}^{3}\left[R_{n \kappa}^{+}+R_{n \kappa}^{-}\right] \text {for } \operatorname{Im} \varepsilon^{(\mathrm{L})} \equiv 0
$$

was investigated. Fig. 8 (a) shows the dependence of the energy balance error $W^{\text {(Error) }}\left(a_{\kappa}^{\text {inc }}\right)$ and of the excitation frequency $\kappa=\operatorname{Re} \kappa_{3}\left(a_{\kappa}^{\text {inc }}\right) / 3$ on the amplitude $a_{\kappa}^{\text {inc }}$ of the incident field, cf. algorithm (9).

The results of calculations showed that, in the studied range of the problem parameters, the absolute value $\left|W^{(\text {Error })}\right|<2.2236 \cdot 10^{-8}$ is acceptable, see Fig. 8 (a).

Fig. 8 (b) illustrates the estimates of the conditioning characteristics of the matrices of dimension 43 obtained in (9) using the composite Weddle's quadrature formula. The estimation of the matrix conditionality was implemented on the basis of the methodology described in [15].

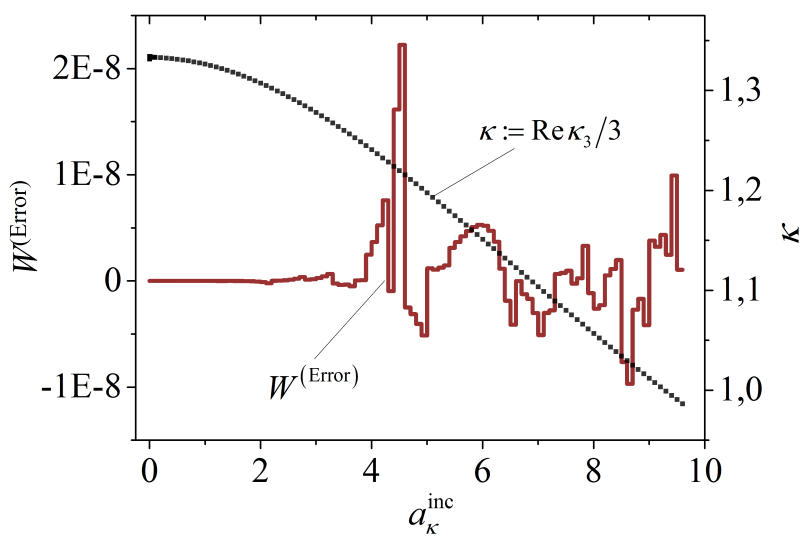

a

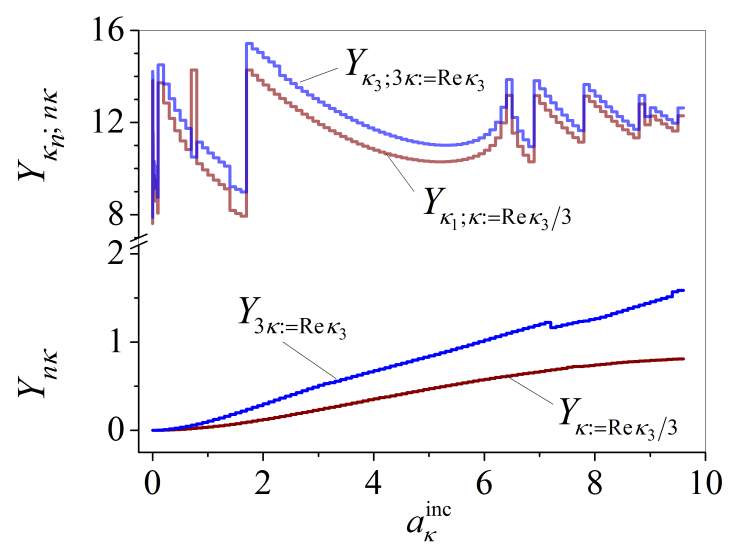

b

Figure 8: Behaviour of the self-consistent iterative processes (9) at $\kappa=\operatorname{Re} \kappa_{3} / 3$ obtained by means of Weddle's quadrature rule for equation systems of dimension $N=43$. Curves: (a) $W^{\text {(Error) }}\left(a_{\kappa}^{\text {inc }}\right)$ the error of the energy balance and $\quad \kappa=\operatorname{Re} \kappa_{3}\left(a_{\kappa}^{\text {inc }}\right) / 3$ the excitation frequencies; (b) $Y_{\kappa_{n} ; n \kappa}\left(a_{\kappa}^{\text {inc }}\right)$ the singularity measure of the matrices of the eigenvalue problems and $Y_{n \kappa}\left(a_{\kappa}^{\text {inc }}\right)$ the sensitiveness of the self-consistent analysis to errors for $n=1,3$.

The degeneration level of the spectral matrices 
$\mathbf{I}-\mathbf{B}_{n \kappa}\left(\kappa_{n}\right)$ at the points of the eigenvalues $\kappa_{n} \in \Omega_{n \kappa} \subset \mathrm{H}_{n \kappa}, n=1,3$, see block 2 in algorithm (9), can be characterized by the quantities

$$
Y_{\kappa_{n} ; n \kappa}=\lg \left\{\operatorname{cond}\left[\mathbf{I}-\mathbf{B}_{n \kappa}\left(\kappa_{n}\right)\right]\right\}, n=1,3 .
$$

The graphs of $Y_{\kappa_{n} ; n \kappa}\left(a_{\kappa}^{\text {inc }}\right), n=1,3$, in dependence on the parameters of the spectral problems are shown in Fig. 8 (b). These values reflect large values of the matrix conditionality, which is an indirect feature of the degeneration (or singularity) of these matrices.

The sensitiveness of the matrices $\mathbf{I}-\mathbf{B}_{n \kappa}$, see block 2 in the algorithm (9), w.r.t. the errors at the scattering $\kappa=\operatorname{Re} \kappa_{3} / 3$ and generation $3 \kappa$ frequencies can be described by the quantities

$$
Y_{n \kappa}=\lg \left\{\operatorname{cond}\left[\mathbf{I}-\mathbf{B}_{n \kappa}\right]\right\}, n=1,3 .
$$

The logarithm of the matrix conditionality gives some information about how many significant digits are lost in solving the system [15].

A discussion of the curves $Y_{n \kappa}\left(a_{\kappa}^{\text {inc }}\right), n=1,3$, depicted in Fig. 8 (b) shows that in the studied parameter range of the self-consistent problem no more than two significant digits are lost.

\section{Conclusions}

The paper describes an iterative algorithm for analyzing the processes of resonant wave radiation by nonlinear layered structures. It is based on the self-consistent solution of nonlinear problems at multiple real frequencies close to complex eigenfrequencies of linearized eigenvalue problems with induced dielectric coefficients.

Results of numerical studies of the properties of scattering and resonant generation of waves by an absolutely transparent layer in the linear approximation are presented. The effect of energy conversion of the incident field into the resonant transmitted field is described. In particular, using the concept of the relative quality factor it was shown that the energy exchange processes occurring during the generation of waves by nonlinear strucures can be indirectly analyzed through the consideration of the spectral characteristics of the structures induced by the incident field.

The mathematical model of the proposed approach is based on a rigorous formulation and a self-consistent solution of a system of nonlinear inhomogeneous and linearized homogeneous spectral boundary value problems of Electrodynamics.

The reliability of the approach and the obtained results obtained are controlled by the following criteria: Verification of an energy balance law, check of the degeneracy (by means of the magnitude of the condition numbers) of the matrices of the eigenvalue problems, confirmation of the low sensitivity to errors of the matrices in the self-consistent analysis of the processes under study (also by means of the condition numbers).

The proposed mathematical model can prove to be very effective in the synthesis of material and geometric parameters of nonlinear structures with optimal resonant properties of scattering, generation, and energy storage.

\section{References}

[1] Y.R. Shen, The Principles of Nonlinear Optics, John Wiley and Sons, New York-Chicester-BrisbaneToronto-Singapore, 1984.

[2] H.M. Gibbs, Optical Bistability: Controlling Light with Light, Academic Press, New York, 1985.

[3] P.H. Siegel, Terahertz technology, IEEE Transactions on Microwave Theory and Techniques 50 (3): 910-928, 2002.

[4] V.K. Miloslavsky, A. Nahal, L.A. Ageev, Peculiarities of spontaneous grating formation in photosensitive films under linearly and circularly polarized radiation, Optics Communications 147: 436-442, 1998.

[5] N.N. Akhmediev, Spatial solitons in Kerr and Kerr-like media, Optical and Quantum Electronics 30: 535-569, 1998.

[6] K. Reimann, Table-top sources of ultrashort $\mathrm{THz}$ pulses, Rep. Prog. Phys. 70: 1597-1632, 2007.

[7] Y.G. Smirnov, D.V. Valovik, Guided electromagnetic waves propagating in a plane dielectric waveguide with nonlinear permittivity, Physical Review A 91 (1): 013840, 2015.

[8] V.V. Yatsyk, Inverse Problems and Large-Scale Computations. Springer, New York, Heidelberg, Dordrecht, London, pp. 199-217, 2013.

[9] L. Angermann, V.V. Yatsyk, The Multifunctional Process of Resonance Scattering and Generation of Oscillations by Nonlinear Layered Structures, Cogent Physics 3 (1): 1-19, 2016.

[10] L. Angermann, V.V. Yatsyk, M.V. Yatsyk, About One Approach to the Analysis of Resonant Scattering and Wave Generation by Nonlinear Layered and Periodic Structures, Phys. Bases of Instrumentation $6(1)$ : pp. 106-117, 2017.

[11]L. Angermann, V.F. Kravchenko, V.I. Pustovoyt, V.V. Yatsyk, Resonance scattering and generation of oscillations by channeling nonlinear layered media, Doklady Physics 58: 535-539, 2013.

[12]L. Angermann, V.V. Yatsyk, M.V. Yatsyk, Methods for analyzing the scattering and generation processes of waves by nonlinear layered media, Phys. Bases of Instrumentation 6 (4): 48-57, 2017.

[13]L. Angermann, V.V. Yatsyk, Resonant Scattering and Generation of Waves: Cubically Polarizable Layers, Springer, Cham, Switzerland, 2019.

[14] V.P. Shestopalov, Y.K. Sirenko, Dynamic Theory of Gratings, Naukova Dumka, Kiev, 1989.

[15]D. Kahaner, C. Moler, S. Nash, Numerical Methods and Software, Prentice-Hall Inc, New Jersey, 1989. 